\title{
Update on Lupus Nephritis: Looking for a New Vision
}

\author{
Enrique Morales ${ }^{a, b}$ Maria Galindo ${ }^{b, c}$ Hernando Trujillo ${ }^{a, b}$ Manuel Praga ${ }^{a, b}, d$

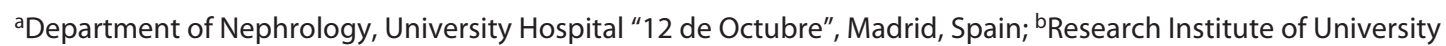 \\ Hospital "12 de Octubre" (imas12), Madrid, Spain; 'Department of Rheumatology, University Hospital "12 de \\ Octubre", Madrid, Spain; 'Department of Medicine, Complutense University of Madrid, Madrid, Spain
}

\section{Keywords}

Lupus nephritis · Biomarkers · Repeat biopsy · Biologics ·

Pregnancy $\cdot$ Kidney transplant

\begin{abstract}
Lupus nephritis (LN) is one of the most common manifestations of systemic lupus erythematosus (SLE), affecting approximately $40 \%$ of patients with lupus. It represents a major risk factor for morbidity and mortality, and $10 \%$ of patients with LN will develop end-stage kidney disease (ESKD). Therefore, there are a number of areas for improvement in the field of LN such as the search for new clinical biomarkers with a more accurate correlation with lupus activity and the redefinition of the histological classification into different subgroups in order to guide a personalized treatment. Although the role of protocol repeat kidney biopsies in LN is controversial, recent publications suggest that repeat histological assessment can be useful in guiding therapeutic decisions that may yield toward precision medicine. In the last decade, LN therapy has remained largely unchanged, with a probability of achieving complete or partial remission not exceeding $60-70 \%$. Thus, optimization of old treatment strategies and search for new agents are urgently needed in order to
\end{abstract}

improve outcomes such as mortality or development of ESKD. Future trials should focus in addressing unanswered issues such as the appropriate dose and duration of immunosuppressive treatment, timing of steroid withdrawal, and drug toxicity. In addition, data are still lacking regarding pregnancy and kidney transplantation in LN and knowledge about these important areas is essential for the management of a subset of patients with SLE. In summary, several major gaps are still present in the therapeutic approach and follow-up of patients with LN. The development of new clinical trial designs will be crucial in the search to improve longterm outcomes.

๑) 2020 S. Karger AG, Basel

\section{Introduction}

Systemic lupus erythematosus (SLE) is a chronic autoimmune disease with immune complex deposits and inflammatory cell infiltration in different tissues of the body, including the kidney. Lupus nephritis (LN) affects a substantial number of SLE patients, representing a major cause of morbidity and mortality. SLE is usually diagnosed in young women in the third decade of life and

$\begin{aligned} & \text { karger@karger.com } \\ & \text { www.karger.com/nef }\end{aligned}$
Karger ${ }^{\prime /}$


represents the leading cause of systemic disease with secondary kidney involvement $[1,2]$. Until recently, the pathological classification of LN focused on the degree of glomerular involvement; however, recent publications have addressed the value of vascular and tubulointerstitial changes, as well as the role of activity and chronicity indices in the prognosis of kidney outcomes [3]. Immunosuppressive therapy is reserved for cases with aggressive histological presentation with the aim of reducing inflammation, stabilizing or improving kidney function, and ultimately achieving clinical remission. However, despite a notable improvement in kidney survival of LN patients in the 1980s and 1990s, the incidence of ESKD in recent decades has only managed to stabilize $[4,5]$. The clinical care of $\mathrm{LN}$ patients includes several aspects that require an individualized approach such as controlling disease activity, decreasing the incidence of flares, avoiding drug toxicity, and achieving a good quality of life. To date, there are important gaps in each of the latter issues that are still subject to significant improvement. These unresolved topics include the following [1]: Do we currently have a prognostic score for our LN patients? [2] What is the role of biomarkers in LN? [3] Is a new perspective on LN pathological classification necessary? [4] How long should we maintain immunosuppressive treatment? [5] How can we manage to reduce adverse effects of immunosuppressive drugs? [6] What is the current situation in special situations such as pregnancy or kidney transplantation in patients with LN? [7] What is the future of LN? In this review, we will try to answer some of these questions with the best available evidence and try to deliver a new vision of LN.

\section{Current Situation of Biomarkers on LN}

Research on biomarkers in LN has produced a large number of publications, yet the same urinary and serum biochemical markers have continued to be used in clinical practice for over 3 decades. Currently, no biomarker has an adequate correlation between clinical manifestations and actual histological damage. This is partly due to the fact that most evidence comes from single-center cohorts with different measurement and definition systems without subsequent validation in multicenter cohorts.

Traditional biomarkers include serum creatinine, hematuria, and proteinuria, with the latter being strongly associated with long-term kidney prognosis $[6,7]$. The predictability of other immunological markers as antidouble stranded DNA (anti-dsDNA) antibodies or com- plement $\mathrm{C} 3$ and $\mathrm{C} 4$ fractions is highly variable, with sensitivities ranging from 50 to $80 \%$ and specificities of around $75 \%[8,9]$.

Certain polymorphisms linked to the major histocompatibility complex such as FcgR IIA, FcgR IIIA, MCP-1, and ITGAM have been associated with LN $[10,11]$; however, they are indicators of susceptibility rather than practical biomarkers for the assessment of disease activity since they are not influenced by treatment. Modifications in the methylation of certain genes [10] as well as circulating microRNAs [12] have also been linked to the development of LN. On the other hand, potential biomarkers not yet incorporated into routine clinical practice include circulating levels of BAFF, APRIL, MBL, soluble IL-7 receptor, cystatin C, and IL-18 $[13,14]$ as well as urinary biomarkers that reflect the state of kidney damage in real time such as microRNAs and levels of MCP-1, TWEAK, NGAL, VCAM-1, and BAFF $[15,16]$. An interesting approach in the future would be the combination of novel and traditional biomarkers in order to identify different activity patterns that allow an individual assessment of patients with LN.

\section{Role of Kidney Biopsy in LN}

Although most cases of LN are represented by glomerulonephritis secondary to deposition of IC, it is important to consider that the clinical and pathological spectrum of $\mathrm{LN}$ is highly heterogeneous and includes entities such as lupus podocytopathy (LP) and thrombotic microangiopathy [17]. Thus, kidney biopsy plays a key role in the diagnosis and management of LN.

The main strengths of the current International Society of Nephrology/Renal Pathology Society (ISN/RPS) LN classification include its wide acceptance by all scientific societies and its practical application regarding therapeutic approach [18]. Nonetheless, it has some limitations such as the absence of clear histological distinction between class III and class IV and the lack of consideration of tubulointerstitial lesions and vascular injury (extension and composition of tubulointerstitial deposits, tubular atrophy, thrombotic microangiopathy, antiphospholipid antibody nephropathy, and lupus vasculopathy) $[19,20]$. Moreover, the recent 2018 classification has removed the IV-S and IV-G subdivisions of class IV due to the inherent difficulty in distinguishing segmental from global lesions depending on the area of the tissue section. On the other hand, the semiquantitative assessment of activity and chronicity indices is now mandatory owing to its prognostic implications [3]. 
The prevalence of the different pathological classes most certainly reflects a clinical selection bias since kidney biopsy is rarely performed in patients without evident signs of kidney disease or in patients with ESKD [21]. A recent study that compared pathological findings of 300 patients with SLE to a group of 560 patients with glomerular diseases secondary to immune complex deposition found that most cases of LN had at least 2 of the following 5 characteristics: (1) "full-house" deposits by immunofluorescence; (2) >2 + staining intensity for Clq; (3) extraglomerular deposits; (4) presence of subendothelial and subepithelial deposits; and (5) endothelial tubularreticular inclusions. The presence of at least 2 of these features had a sensitivity of $92 \%$ and a specificity of $89 \%$ for diagnosis of LN, while the presence of at least 3 of the 5 characteristics had a sensitivity of $80 \%$ and specificity of $95 \%[22]$.

In recent years, several studies have suggested per-protocol repeat biopsies as a valuable source of information in the follow-up of patients with LN. Pathological findings in repeat biopsies could allow determination of a prespecified subsequent therapy [23]. In the Lupus Flares and Histological Renal Activity at the End of Treatment study, maintenance immunosuppression was tapered off after a second kidney biopsy in patients in complete clinical remission for at least 12 months who had received at least 36 months of immunosuppression. Ten of the 11 patients who developed a flare had residual activity on the second kidney biopsy, and all patients with an activity index of $>2$ relapsed. On the other hand, the authors suggested that in patients with an activity index of 0 (complete histological remission), withdrawal of immunosuppression may be reasonable [24]. In another study, serial kidney biopsies were performed in a cohort of 75 patients with $\mathrm{LN}$ who had been under immunosuppressive treatment for 42 months. Maintenance therapy was withdrawn if the biopsy showed an activity index of 0 , but was continued if the biopsy showed an activity index of 1 or more. Based on this protocol, a lower flare rate $(9.2 \%)$ and better kidney outcomes (development of CKD [16\%] or ESKD [0\%]) were observed as compared to those described in previous series [25].

One of the most important efforts by the lupus nephropathy research groups is the search for techniques that overcome interobserver variability among nephropathologists in order to improve characterization of LN lesions, which could alter prognosis and treatment decisions. In addition to the modifications proposed by the recent review of the ISN/RPS [3], visual estimation of histological features can be strengthened by application of digital image analysis techniques. The latter could potentially enable high-throughput, accurate, and reproducible assessment of digitized microscopic images of kidney tissue sections. In fact, recent studies in the fields of diabetic nephropathy [26] and kidney transplantation [27] have shown that digital processing of renal tissue may improve diagnostic profitability and provide useful information that may upgrade traditional diagnostic tools.

Moving forward, a novel pathological classification for LN with prognostic and treatment implications, such as the Oxford for IgA nephropathy, would be desirable in the following years. In addition, application of genomics and new biomarkers in kidney histology could further refine personalized treatment strategies in LN. A proposed algorithm for follow-up based on pathological, clinical, and biochemical parameters is presented in Figure 1.

\section{Role of Biological Therapy in LN}

Different mechanisms have been implicated in the pathogenesis of LN, which justify the study and application of biological therapy. The glomerular damage that occurs in LN is mediated by the formation of immune complexes (IC) at the expense mainly of anti-dsDNA antibodies. However, besides IC, anti-dsDNA antibodies can directly bind to diverse components of the glomerular basement membrane, mesangium, and subendothelial and subepithelial compartments. This results in activation of the complement system, especially the classical pathway, with generation of $\mathrm{C} 3 \mathrm{a}$ and $\mathrm{C} 5 \mathrm{a}$ that have the ability to attract neutrophils and mononuclear cells [28]. Finally, IC can activate other components of the inflammatory response such as the expression of adhesion molecules in the endothelium, favoring the recruitment of proinflammatory leukocytes. The activation of glomerular cells, macrophages, and T cells leads to the production of inflammatory cytokines such as tumor necrosis factor (TNF)-alpha, interleukin (IL)-6, transforming growth factor beta (TGF- $\beta$ ), interferon gamma (IFN- $\gamma$ ), chemokines, and growth factors. All these soluble mediators amplify kidney damage.

Currently, the use of biologics in patients with LN is relegated to severe or refractory cases; however, none has been approved specifically for LN management. Although possible benefits have been described in case series and retrospective studies, efficacy has not been confirmed in controlled clinical trials. Therefore, the use of biological therapies in $\mathrm{LN}$ is still uncertain. 




Fig. 1. Proposed algorithm for monitoring histological and clinical response in LN and for follow-up of patients with LN. The use of clinical, biochemical, and immunological biomarkers together with histological activity can be of great value in determining the risk of disease progression and planning the duration of treatment. A repeat biopsy can provide important information not only in patients with renal flare or persistent proteinuria but also during suspected clinical remission in order to optimize treatment management. AI, activity index; BILAG, British Isles Lupus Assessment Group; CI, chronicity index; IFTA, interstitial fibrosis and tubular atrophy; ISN/RPS, International Society of Nephrology/ Renal Pathology Society; GFR, glomerular filtration rate; LN, lupus nephritis; SCr, serum creatinine; SLEDAI, Systemic Lupus Erythematosus Disease Activity Index.
According to the current EULAR recommendations, biological therapy is reserved for patients who persist with disease activity despite the standard treatment or in cases where it is not possible to taper off steroids [29]. Nonetheless, the use of biologics in LN can be useful from other perspectives such as induction therapy, steroid sparing, or maintenance of remission.

B cells play a key role in the pathogenesis of $\mathrm{LN}$ by producing autoantibodies, secreting cytokines, presenting antigens to $\mathrm{T}$ cells, and modulating dendritic cells. Consequently, direct inhibition of B cells is one of the main objectives of biological therapy.

In fact, most of the published experience comes from biologics targeting B cells [30]. Early data on the efficacy of rituximab in LN were described mainly in patients with refractory disease and originated from case series and retrospective studies [31,32]. These encouraging results led to a prospective randomized placebo-controlled study in patients with LN, the LUNAR trial [33]. Interestingly, although a positive effect of rituximab on isolated parameters such as serological activity and proteinuria was observed, the main objective of $20 \%$ improvement in kidney damage parameters was not achieved. In addition to the
LUNAR trial, findings of the RITUXILUP study suggest a possible role of rituximab as a steroid-sparing agent [34]. B-cell blocking attempts have included different rituximab regimens such as the lymphoma protocol or combination of rituximab with belimumab, while ongoing clinical trials are actively testing other anti-CD20 and anti-CD19 agents [35].

The combination of $\mathrm{B}$-cell depletion with rituximab and inhibition of B-cell survival with belimumab is based on the premise that the production of BAFF following Bcell depletion could facilitate the maturation of autoreactive B cells. However, no benefit on kidney outcomes has been demonstrated with a strategy of induction therapy with rituximab plus cyclophosphamide and subsequent maintenance with belimumab as compared to the standard of care [36]. Thus, more evidence is needed regarding the possible utility of combination strategies in patients with LN.

Fully humanized type I (ocrelizumab and ofatumumab) and type II antibodies (obinutuzumab) represent novel anti-CD20 antibodies for LN management. Although ocrelizumab seemed to be effective in the BELONG study (especially in rituximab-refractory cases), 
the trial was stopped early due to safety concerns [37]. Ofatumumab showed positive effects in a small case series [38]; however, more evidence is needed before considering ofatumumab use in routine clinical practice. Regarding obinutuzumab [39], an ongoing randomized clinical trial is currently testing its potential role and safety for the treatment of SLE (NCT02550652). Of note, both the LUNAR (rituximab) and BELONG (ocrelizumab) trials found similar improvement in kidney response rates (11$12 \%)$ compared to placebo.

When interpreting results of the abovementioned studies, one must consider several potential biases such as different definitions of disease activity criteria, heterogeneous clinical/demographic characteristics of the study population, different aims/outcomes, and diversity of baseline treatment [40]. Of interest, patients with severe kidney involvement were excluded from the studies that provided the rationale for belimumab use in SLE, yet a pooled post hoc analysis of the phase 3 randomized placebo-controlled BLISS trials found that patients with kidney involvement at baseline had greater kidney organ disease improvement with belimumab than with placebo [41]. Subsequently, the BLISS-LN study was specifically designed to evaluate efficacy of belimumab in patients with biopsy-confirmed active LN. Recently, the results of the phase III clinical trial of belimumab in patients with lupus nephritis have been published. The percentage of patients treated with intravenous belimumab added to standard therapy who achieved primary and secondary efficacy endpoints was significantly higher compared to those treated with standard therapy alone. On the other hand, the risk of renal disease event or death at any time up to week 104 was $49 \%$ lower in the belimumab group vs. placebo group $(\mathrm{HR}=0.51 ; 95 \%$ CI $0.34-0.77, p=$ 0.0014) [42].

In addition to $\mathrm{B}$ cells, activation of self-reactive $\mathrm{T}$ cells is another pathogenic mechanism of LN. These cells require the costimulation signal CD28-CD80/86. Specific blocking of this signal has been shown to be effective in experimental animal models of LN[43]. However, clinical trials with abatacept failed to demonstrate efficacy in LN patients $[44,45]$. Blocking costimulation with abatacept also failed to improve the outcome of LN at either 24 or 52 weeks when added to cyclophosphamide in the induction phase. Nonetheless, it could be an effective alternative in maintaining remission [46].

Anifrolumab, a type I IFN receptor antagonist, reduced disease activity compared to placebo in SLE without kidney involvement [47], and it is currently under investigation in patients with LN (NCT02547922). Of note, anifrolumab and other type I IFN antagonists such as rontalizumab or sifalimumab have shown to be effective only in patients with high expression of the IFN signal [48].

Despite the indisputable pathogenic involvement of the complement system in SLE, few attempts have been made with complement blockers in patients with LN. The use of eculizumab (C5 inhibitor) has been limited to patients with antiphospholipid syndrome (APS) and LN with associated thrombotic microangiopathy [49].

One of the cytokines involved in the pathogenesis of SLE is IL-2, which is essential to maintain the activation and proliferation of $\mathrm{T}$ cells. The characteristic low levels of IL-2 observed in SLE patients may contribute to a reduction in the number of regulatory $\mathrm{T}$ cells and cytotoxic lymphocytes. In a small preliminary study, the administration of low-dose IL-2 demonstrated clinical efficacy and improvement of laboratory parameters such as complement levels, anti-dsDNA antibody titers, and proteinuria [50]. More recently, a prospective controlled trial showed that low-dose IL-2 on top of standard treatment might be effective in achieving remission in patients with LN [51].

Promising preliminary results with ustekinumab (IL12/IL-23 inhibitor) have been reported in SLE patients; however, data on its efficacy in LN are still unknown [52]. In addition, JAK inhibition has shown promise in murine LN, while autologous hematopoietic stem cell transplantation has been suggested as an alternative treatment of refractory LN, though not without controversy [53].

\section{Special Situations (Lupus Podocytopathy, Membranous Nephropathy, and Antiphospoholipid Syndrome)}

LP is a glomerular lesion distinguished by podocyte foot process effacement without immune complex deposition or with minimal mesangial deposition [54]. Overall, the prevalence of LP represents approximately $1 \%$ of all LN biopsies. Proposed criteria for the diagnosis of LP include the following: (1) nephrotic syndrome in a patient with SLE; (2) presence of diffuse podocyte effacement on electron microscopy; and (3) absence of immune subendothelial or subepithelial deposits by immunofluorescence and electron microscopy [55]. Although normal glomeruli are usually observed on light microscopy, lesions of focal segmental glomerulosclerosis (FSGS) with or without mesangial proliferation can also be observed. In the largest cohort of LP published to date $(n=50), 13$ 
cases presented with normal light microscopy, 9 cases with lesions of FSGS, and 28 cases with mesangial proliferation. In addition, 47 out of 50 cases (94\%) exhibited mesangial deposits by immunofluorescence and electron microscopy, while most patients with complete nephrotic syndrome had $>70 \%$ diffuse foot process effacement [56]. In African American patients with LN and FSGS features on kidney histology, one must consider testing for APOL1 G1 and G2 risk alleles due to its association with collapsing glomerulopathy [57]. Regarding treatment of LP, most cases respond to a short cycle of highdose steroids; however, high relapse rates have been reported in a subset of patients. A remission rate of $94 \%$ has been observed with the use of steroids alone or in combination with other immunosuppressants [56]. Nonetheless, LP with FSGS features seems to respond less to steroids. In these particular cases, addition of mycophenolate mofetil, calcineurin inhibitors, or rituximab may be considered.

Membranous nephropathy (MN) or LN class V is a subtype of LN in which IC, by definition, are deposited in the glomerular subepithelial space. Proteinuria with or without complete nephrotic syndrome is the most characteristic feature of $\mathrm{MN}$ [58]. In last years, differentiation between primary and secondary forms has significantly improved thanks to the description of autoantibodies against the podocyte antigen M-type phospholipase A2 receptor (PLA2R). Moreover, a recent work proposed that glomerular proteins exostosin 1/exostosin 2 may represent biomarkers for $\mathrm{MN}$ associated with autoimmune disease [59]. Treatment of class V LN includes supportive measures such as sodium restriction and antiproteinuric agents such as angiotensin-converting enzyme inhibitors (ACEi) or angiotensin receptor blockers (ARBs) [60]. In patients with nephrotic syndrome, treatment should also include statins for dyslipidemia, hypoproteic diet, and prophylactic anticoagulation based on individual's bleeding risk profile. Because of the lack of well-designed controlled clinical trials, the optimal specific treatment for membranous LN remains unclear. In a recent meta-analysis, no significant differences were found in response to treatment according to the choice of immunosuppressant (azathioprine $88 \%$, cyclophosphamide $75 \%$, and mycophenolate mofetil and cyclosporine 84\%) [61]. Therefore, future clinical trials are necessary to provide an answer regarding first-line immunosuppression choice in class V LN.

APS is an autoimmune disease characterized by recurrent thrombotic events and/or pregnancy morbidity associated with the presence of antiphospholipid antibodies
(aPL) [62]. APS may be primary or secondary. The latter occurs in the context of underlying autoimmune disease such as SLE. aPL are detected in $30-50 \%$ of SLE patients, sometimes associated with thromboembolic complications. The actual prevalence of kidney involvement in APS is difficult to determine due to the scarcity of published data and because an exhaustive search for vascular lesions in kidney biopsies is not always performed. Kidney involvement ranges from large vessel lesions (renal vein thrombosis, renal artery stenosis, renal infarction, and allograft vascular thrombosis) to involvement of small intrarenal vessels [63]. The latter is known as APSassociated nephropathy (APSN) and includes acute lesions with fibrin thrombus formation in glomerular capillaries or arterioles (thrombotic microangiopathy) and nonspecific chronic lesions such as arteriosclerosis, recanalized thrombi, fibrous intimal hyperplasia, tubular thyroidization, and focal cortical atrophy. Much less often, glomerular involvement has been described in the forms of $\mathrm{MN}$, mesangial $\mathrm{C} 3$, or segmental glomerular sclerosis [64]. Clinically, primary APS is characterized by hypertension, proteinuria, hematuria, or kidney failure [65]. It is important to point out that vascular lesions observed in APS may also occur in patients with LN; thus, thorough pathological assessment is crucial in order to detect them. Little information has been published regarding APSN in SLE patients. Besides, reports have not been particularly consistent [66]. According to diverse studies, the prevalence of APSN in SLE is highly variable, ranging between 11 and 50\% depending on the series and the definition criteria. Despite the fact that vascular injuries are clearly associated with a worse functional prognosis in LN, they are often not given much consideration [67]. Acute vascular lesions are infrequently observed with routine histological techniques given its low prevalence or because of inadequate sample size. On the other hand, chronic vascular lesions are nonspecific and may be related to age or cardiovascular risk factors associated with arteriosclerosis. Interestingly, immunodetection of intravascular CD61 + platelet aggregates has shown to increase sensitivity of microthrombus identification regardless of the presence of aPL. In addition, the presence of microthrombosis has been significantly correlated with higher macrophagic infiltration $[68,69]$. In any case, the detection of vascular involvement in kidney biopsies of LN patients is of great importance since its presence has been associated with a negative impact on kidney outcomes $[67,70]$. Both cellular and soluble elements have been implicated in the pathogenesis of APS. On the one hand, the presence of acute vascular lesions has been re- 
lated to the characteristic macrophage, platelet, and endothelial activation of LN. On the other hand, several mechanisms by which the complement system may contribute to thrombotic events have been suggested. Activation of complement leads to cleavage of $\mathrm{C} 5$, generating $\mathrm{C} 5 \mathrm{a}$ and $\mathrm{C} 5 \mathrm{~b}$ (leading to membrane attack complex formation). Complement activation may drive to neutrophil expression of tissue factor (TF) mediated through the $\mathrm{C} 5 \mathrm{a}$ receptor, leading to expression of procoagulant activity [71]. C5a also induces TF expression on monocytes and endothelial cells [72]. In addition, the deposition of C5b9 on the endothelial surface further amplifies activation of coagulation and thrombosis [73]. Finally, the complement system can also contribute to depressed fibrinolysis in APS [74]. In view of the above, the addition of anticoagulation to standard therapy is recommended in patients with LN and APS [75]. In cases with recurrent thrombosis despite anticoagulation, the association of low-dose aspirin, hydroxychloroquine, or statins may be considered. There is limited information regarding treatment of catastrophic LN-associated APS. However, in this rare scenario, the use of complement inhibitors such as eculizumab, mammalian target of rapamycin inhibitors, anti-B-cell therapy, or plasma exchange may be contemplated.

\section{Kidney Transplantation and LN}

Between 10 and $30 \%$ of patients with SLE and LN will progress to ESKD [76]. Although at present there are no consensus recommendations on the wait time to transplant for patients with LN, ideally, it should be as short as possible. In addition, assessment of the different options for kidney transplantation in these patients (deceased donor and living donor kidney transplantation) must be addressed [76]. The majority of studies, especially the largest and most contemporary ones, have shown that kidney transplant results in patients with SLE are comparable to patients without SLE. Nonetheless, some peculiarities merit special attention during pretransplant evaluation and follow-up. Anti-cardiolipin antibodies have been associated with thrombotic risk, and its presence must be discarded in SLE patients. Also, a detailed assessment of cardiovascular history is of utmost importance due to the intrinsic cardiovascular risk of SLE [77]. Since LN patients are exposed to immunosuppressive treatment throughout their lives, the added exposure after a kidney transplant could increase the risk of neoplasms. Therefore, a closer follow-up is recommended in this regard

Update on Lupus Nephritis
[78]. Diagnosis of posttransplant LN recurrence is not always available given that immunofluorescence and electron microscopy techniques are indispensable [79]. Most kidney transplant recipients receive maintenance immunosuppressive therapy with calcineurin inhibitors, mycophenolate mofetil, and steroids. Notably, this combination (known as multitarget therapy) has been proposed by some groups as an alternative option for induction treatment of LN [80]. It is tempting to speculate that the low incidence of LN recurrence after renal transplantation can be attributed to the use of this specific combination.

\section{Pregnancy and LN}

Physiological changes during pregnancy can lead to impaired kidney function in patients with underlying kidney disease such as LN. In addition, precedent chronic kidney disease increases the risk of developing pre-eclampsia (PE) [81]. In normal pregnancies, serum creatinine levels may decrease to $0.4-0.6 \mathrm{mg} / \mathrm{dL}$ due to an increase (up to $30-50 \%$ ) in glomerular filtration rate. Also, urinary protein excretion increases to values between 0.18 and $0.25 \mathrm{~g} /$ day. However, diagnostic criteria for LN in pregnant women are the same as in nonpregnant patients. The presence of extrarenal activity, positive or increasing titers of anti-dsDNA antibodies, and/or low complement levels should raise suspicion of LN. If reasonable doubts persist despite a thorough clinical and biochemical assessment, a kidney biopsy should be considered if no absolute contraindications exist. Conversely, renal flares during pregnancy in patients with pre-existent biopsy-proven LN are relatively simple to diagnose, especially if they occur before the 20th week of gestation. In these cases, baseline immunosuppressive treatment can be intensified, reserving a repeat kidney biopsy only for those without response.

Patients with SLE have an increased incidence of PE, ranging from 11 to $35 \%$ compared to a $5 \%$ risk in the general population $[82,83]$. Diagnosis is not always straightforward as proteinuria, hypertension, or edema may be present in LN as well as in PE. In the last decade, an impaired balance between angiogenesis markers has been described in the serum of patients with PE compared to healthy pregnant women. These changes include increased soluble fms-like tyrosine kinase 1 (sFlt-1), increased endoglin, decreased placental growth factor (PlGF), and increased sFlt-1/PlGF ratio [84, 85]. Interestingly, recent studies have shown that these markers be- 
have differently in patients with SLE and/or APS compared to PE, implying that its application may be useful for differential diagnosis [86]. On the other hand, Doppler of the uterine arteries has been postulated for many years as a promising method for the prediction of PE [83]. The use of angiogenic indices combined with Doppler of uterine arteries between the 20th and 26th week of gestation has proven to be an accurate predictive means for early PE ( $<34$ weeks) but not for late PE $[87,88]$.

The first step in the event of an LN flare during pregnancy is to start or increase baseline steroid therapy. The standard recommended dose is $0.5-1 \mathrm{mg} / \mathrm{kg} /$ day of prednisone or equivalent. However, evidence from small clinical trials and observational studies in nonpregnant patients suggests that intravenous pulses of methylprednisolone (250-500 mg/day for 3 consecutive days) followed by $20-30 \mathrm{mg} /$ day of oral prednisone or equivalent permit lower total steroid dosage $[89,90]$, with consequent risk reduction in fetal and maternal complications. In addition to steroids, hydroxychloroquine should be started in patients who were not already receiving it. High-quality evidence exists regarding positive effects (decreased lupus activity) of hydroxychloroquine in pregnant women with SLE, without risks of hurting the baby $[91,92]$. Azathioprine is the immunosuppressant of choice for both maintenance treatment of patients with pre-existing LN and induction of newly diagnosed cases [91]. Calcineurin inhibitors (cyclosporine and tacrolimus) have also proven to be safe during pregnancy. Nonetheless, tacrolimus has been associated with an increased risk of PE, gestational diabetes, and hypertension as compared to cyclosporine [81]. Of note, because of an increase in the volume of distribution during pregnancy, calcineurin inhibitors should be started at the minimum effective dose and serum levels should be monitored periodically in order to avoid toxicity [91]. In cases not responding to standard or traditional therapies, addition of intravenous immunoglobulins may be considered [91, 93]. Cyclophosphamide during the first trimester is contraindicated due to its known teratogenic effects [94]. However, in exceptional cases where vital risk for the mother and fetal immaturity are encountered, some authors have suggested cyclophosphamide as a salvage treatment in the second or third trimester since no significant fetal complications have been reported in pregnant patients with breast cancer [91]. Current evidence indicates that the use of rituximab in the first trimester of pregnancy does not increase the risk of fetal malformations. Nevertheless, there is a significant risk of B-cell depletion and cytopenias in the newborn, increasing the risk of infectious events. Thus, rituximab should be used only in exceptional cases [95, 96]. Plasmapheresis or immunoadsorption may be considered as a last option in patients refractory to all previously described therapies [97].

The use of ACEi or ARBs for blood pressure control is contraindicated during pregnancy due to their negative effects on kidney development and fetal growth. These agents should be withdrawn upon pregnancy diagnosis or even before whenever possible [98]. As alternatives, labetalol, nifedipine, methyldopa, or hydralazine can be used, although one must consider that these drugs lack antiproteinuric effect. Diuretics are not recommended in patients with PE or oligoamnios due to the risk of reduced placental flow and intravascular volume depletion, which in pre-eclamptic patients is already decreased compared to normal pregnancy. In addition, patients with a history of LN or evidence of active disease should be on low-dose aspirin (100 mg/day) before week 12 due to the intrinsic high risk of developing PE.

\section{What Is the Optimal Duration of Maintenance Immunosuppressive Therapy in LN?}

There is a lack of common criteria among different scientific societies regarding these issues, and it probably represents one of the most controversial aspects in the area of LN. Current clinical guidelines recommend at least 3 years of treatment including 1 year of complete clinical remission before considering immunosuppression withdrawal. However, in some studies such as the MANTAIN, $>50 \%$ of patients remained under immunosuppressive treatment for over 10 years [99]. The decision of immunosuppression withdrawal is not without risks since flares may lead to worse kidney outcomes and greater morbidity and mortality associated with lupus activity $[100,101]$. Induction therapy entails a relatively short period of high-dose immunosuppression (3-6 months) with increased risks of adverse effects in favor of achieving a high percentage of clinical remission. Conversely, the maintenance period encompasses a longer time of less aggressive immunosuppression with the aim of achieving complete remission while avoiding relapses [102]. However, the low-moderate doses of immunosuppression used during the maintenance period are also associated with toxicity that can lead to poor tolerability and acceptability of treatment. Adverse effects include increased susceptibility to infections, malignancies, bone marrow toxicity, and a significant cost burden on healthcare systems [101]. Many issues should be considered when the 


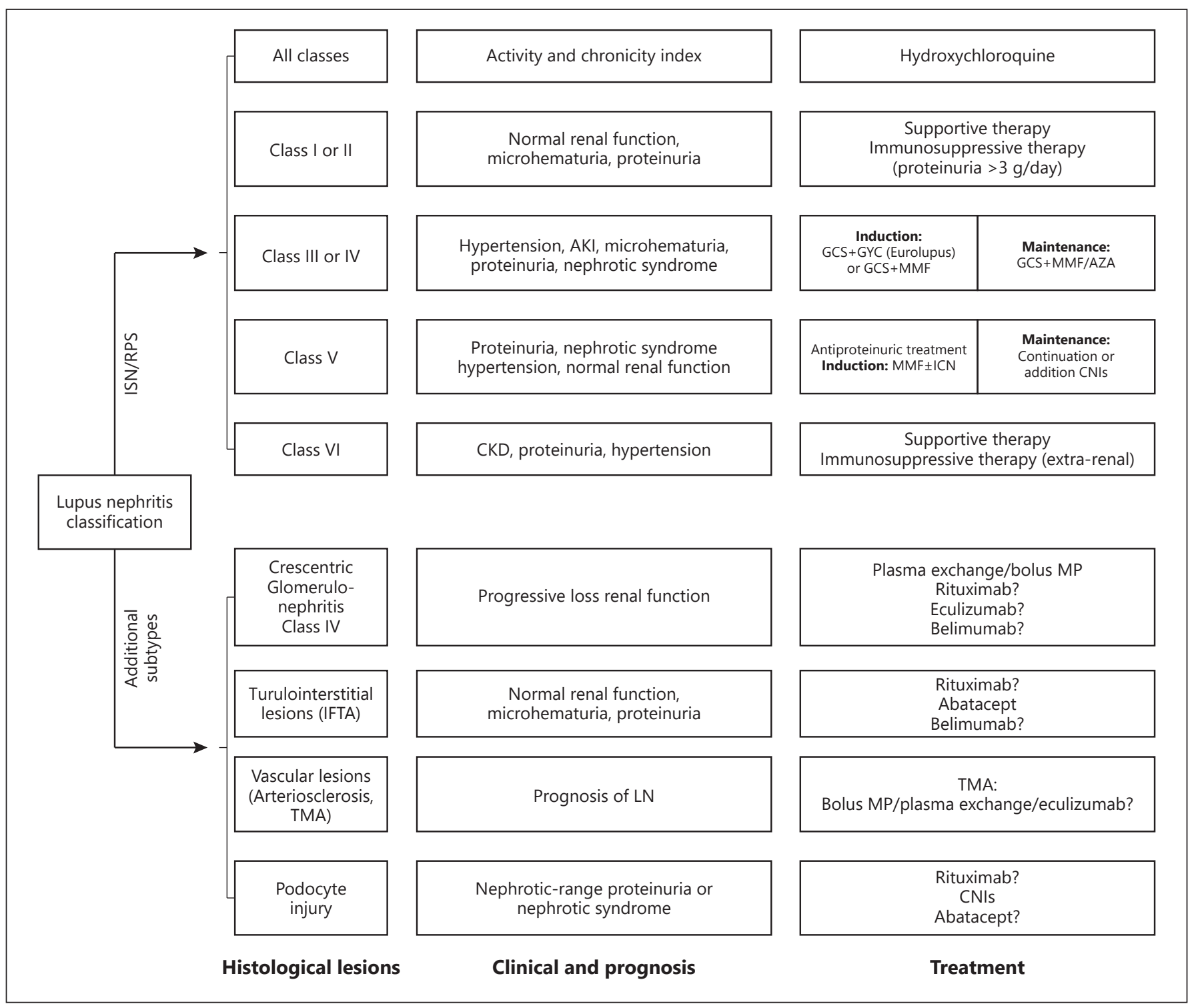

Fig. 2. Proposed treatment algorithm for different pathological subtypes of LN. This treatment proposal combines the classic histological classes of LN defined by the ISN/RPS together with different subtypes that incorporate tubulointerstitial and vascular lesions. These histological phenotypes combined with clinical manifestations and their prognostic implications may establish a more

decision is made to withdraw maintenance treatment: What was the time to achieve complete remission? Has histological remission been achieved? Is there evidence of ongoing extrarenal disease activity? Did the patient have renal flares?

Hydroxychloroquine is essential during the maintenance phase because it is associated with improved response and less relapses as compared to placebo [102]. On personalized management of LN. AKI, acute kidney injury; CYC, cyclophosphamide; CNIs, calcineurin inhibitors; $\mathrm{CKD}$, chronic kidney disease; GCS, glucocorticoids; LN, lupus nephritis; ISN/ RPS, International Society of Nephrology/Renal Pathology Society; MMF, mycophenolate mofetil; TMA, thrombotic microangiopathy.

the contrary, the adequate duration of immunosuppressive drugs in maintenance therapy continues to be a highly controversial aspect [103]. The decision to perform a repeat kidney biopsy has been in the spotlight in recent years. According to some studies, pathological data from repeat kidney biopsies in LN patients could be useful to guide therapeutic approach during the maintenance period $[104,105]$. Controlled prospective trials with extend- 
ed follow-up are urgently needed to determine which patients have higher risk of flares and to better define the appropriate time for immunosuppression withdrawal.

\section{Conclusions and the Future of LN}

The future of LN should be directed toward the search of different phenotypes according to the clinical and histological characteristics of each patient in order to establish a more personalized therapeutic strategy (Fig. 2). In addition, novel serum and histological biomarkers are needed to accurately assess the degree of response and to better define the risk of progression to ESKD. Although biochemical markers such as proteinuria, serum creatinine, and glomerular filtration rate have been traditionally used to evaluate outcomes in LN studies, we believe that future prospective randomized clinical trials should also include histological findings (tubulointerstitial and vascular lesions and activity and chronicity indices) as surrogate endpoints. Data from protocol biopsies could provide interesting insights regarding the optimal time for immunosuppression withdrawal. Finally, the encour- aging preliminary results of several ongoing trials suggest that less toxic novel biological therapies will be available in the near future that will allow our patients to have a better quality of life.

\section{Conflict of Interest Statement}

The authors have no conflicts of interest to declare.

\section{Funding Sources}

E.M. holds a research grant (2017/0122) "Desarrollo de un índice de gravedad en la Nefritis Lúpica" from Fundación Madrileña de Nefrología. M.G. holds a research grant (2017/0030) "Desarrollo de un índice de gravedad en la nefritis lúpica" from Sociedad de Reumatología de la Comunidad de Madrid (SORCOM).

\section{Author Contributions}

All the authors contributed equally to this study, revised the paper, and approved the final version of the manuscript.

\section{References}

1 Dörner T, Furie R. Novel paradigms in systemic lupus erythematosus. Lancet. 2019; 393(10188):2344-58.

2 Almaani S, Meara A, Rovin BH. Update on lupus nephritis. Clin J Am Soc Nephrol. 2017; 12(5):825-35.

3 Bajema IM, Wilhelmus S, Alpers CE, Bruijn JA, Colvin RB, Cook HT, et al. Revision of the International Society of Nephrology/Renal Pathology Society classification for lupus nephritis: clarification of definitions, and modified National Institutes of Health activity and chronicity indices. Kidney Int. 2018;93(4):789-96.

4 Fanouriakis A, Bertsias G. Changing paradigms in the treatment of systemic lupus erythematosus. Lupus Sci Med. 2019;6(1): e000310.

5 Avasare RS, Yee J. Lupus nephritis: breaking the lull. Adv Chronic Kidney Dis. 2019;26(5): 307-10.

6 Dall'Era M, Cisternas MG, Smilek DE, Straub L, Houssiau FA, Cervera R, et al. Predictors of long-term renal outcome in lupus nephritis trials: lessons learned from the Euro-lupus nephritis cohort. Arthritis Rheumatol. 2015; 67(5):1305-13

7 Tamirou F, D'Cruz D, Sangle S, Remy P, Vasconcelos C, Fiehn C, et al. Long-term followup of the MAINTAIN Nephritis Trial, comparing azathioprine and mycophenolate mofetil as maintenance therapy of lupus nephritis. Ann Rheum Dis. 2016;75(3):526-31.
8 Moroni G, Radice A, Giammarresi G, Quaglini S, Gallelli B, Leoni A, et al. Are laboratory tests useful for monitoring the activity of lupus nephritis? A 6-year prospective study in a cohort of 228 patients with lupus nephritis. Ann Rheum Dis. 2009;68(2):234-7.

9 Esdaile JM, Joseph L, Abrahamowicz M, Li Y, Danoff D, Clarke AE. Routine immunologic tests in systemic lupus erythematosus: is there a need for more studies? J Rheumatol. 1996; 23(11):1891-6.

10 Aitman TJ, Dong R, Vyse TJ, Norsworthy PJ, Johnson MD, Smith J, et al. Copy number polymorphism in Fcgr3 predisposes to glomerulonephritis in rats and humans. Nature. 2006;439(7078):851-5.

11 Malafronte P, Vieira JM Jr, Pereira AC, Krieger JE, Barros RT, Woronik V. Association of the MCP-1-2518 A/G polymorphism and no association of its receptor CCR2-64 V/I polymorphism with lupus nephritis. J Rheumatol. 2010;37(4):776-82.

12 Coit P, Renauer P, Jeffries MA, Merrill JT, McCune WJ, Maksimowicz-McKinnon K, et al. Renal involvement in lupus is characterized by unique DNA methylation changes in naive CD4+ T cells. J Autoimmun. 2015;61: 29-35.

13 Ahearn JM, Liu CC, Kao AH, Manzi S. Biomarkers for systemic lupus erythematosus. Transl Res. 2012;159(4):326-42.
14 Treamtrakanpon W, Tantivitayakul P, Benjachat T, Somparn P, KittikowitW, Eiamong S, et al. APRIL, a proliferation-inducing ligand, as a potential marker of lupus nephritis. Arthritis Res Ther. 2012;14(6):R252.

15 Perez-Hernandez J, Forner MJ, Pinto C, Chaves FJ, Cortes R, Redon J. Increased urinary exosomal microRNAs in patients with systemic lupus erythematosus. PLoS One. 2015;10(9):e0138618.

16 Schwartz N, Michaelson JS, Putterman C. Lipocalin-2, TWEAK, and other cytokines as urinary biomarkers for lupus nephritis. Ann N Y Acad Sci. 2007;1109:265-74.

17 Stokes MB, D'Agati VD. Classification of lupus nephritis; time for a change? Adv Chronic Kidney Dis. 2019;26(5):323-9.

18 Weening JJ, D’Agati VD, Schwartz MM, Seshan SV, Alpers CE, Appel GB, et al. International Society of Nephrology working group on the classification of lupus nephritis; renal pathology society working group on the classification of lupus nephritis. The classification of glomerulonephritis in systemic lupus erythematosus revisited. Kidney Int. 2004;65(2):521-30.

19 Leatherwood C, Speyer CB, Feldman CH, D’Silva K, Gómez-Puerta JA, Hoover PJ, et al. Clinical characteristics and renal prognosis associated with interstitial fibrosis and tubular atrophy (IFTA) and vascular injury in lupus nephritis biopsies. Semin Arthritis Rheum. 2019;49(3):396-404. 
20 Yu F, Haas M, Glassock R, Zhao MH. Redefining lupus nephritis: clinical implications of pathophysiologic subtypes. Nat Rev Nephrol. 2017;13(8):483-95.

21 Ayoub I, Cassol C, Almaani S, Rovin B, Parikh SV. The kidney biopsy in systemic lupus erythematosus: a view of the past and a vision of the future. Adv Chronic Kidney Dis. 2019; 26(5):360-8.

22 Kudose S, Santoriello D, Bomback AS, Stokes MB, D'Agati VD, Markowitz GS. Sensitivity and specificity of pathologic findings to diagnose lupus nephritis. Clin J Am Soc Nephrol. 2019;14(11):1605-15.

23 Nachman PH. Repeat kidney biopsy for lupus nephritis: an important step forward. Kidney Int. 2018;94(4):659-61.

24 De Rosa M, Azzato F, Toblli JE, De Rosa G, Fuentes F, Nagaraja HN, et al. A prospective observational cohort study highlights kidney biopsy findings of lupus nephritis patients in remission who flare following withdrawal of maintenance therapy. Kidney Int. 2018;94(4): 788-94.

25 Malvar A, Alberton V, Lococo B, Ferrari M, Delgado P, Nagaraja HN, et al. Kidney biopsy-based management of maintenance immunosuppression is safe and may ameliorate flare rate in lupus nephritis. Kidney Int. 2020; 97(1):156-62.

26 Ginley B, Lutnick B, Jen KY, Fogo AB, Jain S, Rosenberg A, et al. Computational segmentation and classification of diabetic glomerulosclerosis. J Am Soc Nephrol. 2019;30(10): 1953-67.

27 Hermsen M, de Bel T, den Boer M, Steenbergen EJ, Kers J, Florquin S, et al. Deep learning-based histopathologic assessment of kidney tissue. J Am Soc Nephrol. 2019;30(10):1968-79.

28 Lech M, Anders HJ. The pathogenesis of lupus nephritis. J Am Soc Nephrol. 2013;24(9): 1357-66.

29 Fanouriakis A, Kostopoulou M, Alunno A, Aringer M, Bajema I, Boletis JN, et al. 2019 update of the EULAR recommendations for the management of systemic lupus erythematosus. Ann Rheum Dis. 2019;78(6):736-45.

30 Almaani S, Rovin BH. B-cell therapy in lupus nephritis: an overview. Nephrol Dial Transplant. 2019;34(1):22-9.

31 Fernández-Nebro A, de la Fuente JL, Carreño L, Izquierdo MG, Tomero E, Rúa-Figueroa I, et al. Multicenter longitudinal study of B-lymphocyte depletion in refractory systemic lupus erythematosus: the LESIMAB study. Lupus. 2012;21(10):1063-76.

32 Iaccarino L, Bartoloni E, Carli L, Ceccarelli F, Conti F, De Vita S, et al. Efficacy and safety of off-label use of rituximab in refractory lupus: data from the Italian multicentre registry. Clin Exp Rheumatol. 2015;33(4):449-56.

33 Rovin BH, Furie R, Latinis K, Looney RJ, Fervenza FC, Sanchez-Guerrero J, et al. Efficacy and safety of rituximab in patients with active proliferative lupus nephritis: the lupus nephritis assessment with rituximab study. Arthritis Rheum. 2012;64(4):1215.
34 Condon MB, Ashby D, Pepper RJ, Cook HT, Levy JB, Griffith M, et al. Prospective observational single-centre cohort study to evaluate the effectiveness of treating lupus nephritis with rituximab and mycophenolate mofetil but no oral steroids. Ann Rheum Dis. 2013; 72(8):1280-6

35 Haarhaus ML, Svenungsson E, Gunnarsson I. Ofatumumab treatment in lupus nephritis patients. Clin Kidney J. 2016;9(4):552-5.

36 Kraaij T, Huizinga TW, Rabelink TJ, Teng YK. Belimumab after rituximab as maintenance therapy in lupus nephritis. Rheumatology. 2014;53(11):2122-4.

37 Mysler EF, Spindler AJ, Guzman R, Bijl M, Jayne D, Furie RA, et al. Efficacy and safety of ocrelizumab in active proliferative lupus nephritis: results from a randomized, doubleblind, phase III study. Arthritis Rheum. 2013; 65(9):2368-79.

38 Haarhaus ML, Svenungsson E, Gunnarsson I. Ofatumumab treatment in lupus nephritis patients. Clin Kidney J. 2016;9(4):552-5.

39 Reddy V, Dahal LN, Cragg MS, Glennie MJ, Cambridge G, Cragg MS, et al. Optimizing Bcell depletion in autoimmune disease: is obinutuzumab the answer? Drug Discov Today. 2016;21(8):1330-8.

40 Hruskova Z, Tesar V. Lessons learned from the failure of several recent trials with biologic treatment in systemic lupus erythematosus. Expert Opin Biol Ther. 2018;18(9):989-96.

41 Dooley MA, Houssiau F, Aranow C, D'Cruz DP, Askanase A, Roth DA, et al. Effect of belimumab treatment on renal outcomes: results from the phase 3 belimumab clinical trials in patients with SLE. Lupus. 2013;22(1): 63-72.

42 Furie R, Rovin BH, Houssiau F, Malvar A, Teng YKO, Contreras G, et al. Two-year, randomized, controlled trial of belimumab in lupus nephritis. N Engl J Med. 2020;383(12): 1117-28.

43 Schiffer L, Sinha J, Wang X, Huang W, von Gersdorff G, Schiffer M, et al. Short term administration of costimulatory blockade and cyclophosphamide induces remission of systemic lupus erythematosus nephritis in NZB/W F1 mice by a mechanism downstream of renal immune complex deposition. J Immunol. 2003;171(1):489-97.

44 Furie R, Nicholls K, Cheng TT, Houssiau F, Burgos-Vargas R, Chen SL, et al. Efficacy and safety of abatacept in lupus nephritis: a twelve-month, randomized, double-blind study. Arthritis Rheumatol. 2014;66(2):37989.

45 Group AT. Treatment of lupus nephritis with abatacept: the abatacept and cyclophosphamide combination efficacy and safety study. Arthritis Rheum. 2014;66(11): 3096-104.

46 ACCESS Trial Group. Treatment of lupus nephritis with abatacept: the abatacept and cyclophosphamide combination efficacy and safety study. Arthritis Rheum. 2014;66(11): 3096-104.
47 Furie R, Khamashta M, Merrill JT, Werth VP, Kalunian K, Brohawn P, et al. Anifrolumab, an anti-interferon- $\alpha$ receptor monoclonal antibody, in moderate-to-severe systemic lupus erythematosus. Arthritis Rheumatol. 2017; 69(2):376-86.

48 Khamashta M, Merrill JT, Werth VP, Furie R Kalunian K, Illei GG, et al. Sifalimumab, an anti-interferon-alpha monoclonal antibody, in moderate to severe systemic lupus erythematosus: a randomised, double-blind, placebo-controlled study. Ann Rheum Dis. 2016; 75(11):1909-16.

49 Sciascia S, Radin M, Yazdany J, Tektonidou M, Cecchi I, Roccatello D, et al. Expanding the therapeutic options for renal involvement in lupus: eculizumab, available evidence. Rheumatol Int. 2017;37(8):1249-55.

50 Shao M, He J, Zhang R, Zhang X, Yang Y, Li $\mathrm{C}$, et al. Interleukin-2 deficiency associated with renal impairment in systemic lupus erythematosus. J Interferon Cytokine Res. 2019; 39(2):117-24.

51 He J, Zhang R, Shao M, Zhao X, Miao M, Chen J, et al. Efficacy and safety of low-dose IL-2 in the treatment of systemic lupus erythematosus: a randomised, double-blind, placebo-controlled trial. Ann Rheum Dis. 2020; 79(1):141-9.

52 Parikh SV, Rovin BH. Current and emerging therapies for lupus nephritis. J Am Soc Nephrol. 2016;27(10):2929-39.

53 Huang X, Chen W, Ren G, Zhao L, Guo J, Gong D, et al. Autologous hematopoietic stem cell transplantation for refractory lupus nephritis. Clin J Am Soc Nephrol. 2019;14(5): 719-27.

54 Chen D, Hu W. Lupus podocytopathy: a distinct entity of lupus nephritis. J Nephrol. 2018;31(5):629-34.

55 Oliva-Damaso N, Payan J, Oliva-Damaso E, Pereda T, Bomback AS. Lupus podocytopathy: an overview. Adv Chronic Kidney Dis. 2019;26(5):369-75.

$56 \mathrm{Hu}$ W, Chen Y, Wang S, Chen H, Liu Z, Zeng $\mathrm{C}$, et al. Clinical-morphological features and outcomes of lupus podocytopathy. Clin J Am Soc Nephrol. 2016;11(4):585-92.

57 Morales E, Alonso M, Gutiérrez E. Collapsing glomerulopathy: update. Med Clin. 2019; 152(9):361-7.

58 Almaani S, Parikh SV. Membranous lupus nephritis: a clinical review. Adv Chronic Kidney Dis. 2019;26(5):393-403.

59 Sethi S, Madden BJ, Debiec H, Charlesworth MC, Gross L, Ravindran A, et al. Exostosin 1/ exostosin 2-associated membranous nephropathy. J Am Soc Nephrol. 2019;30(6) 1123-36.

60 Morales E, Millet VG, Rojas-Rivera J, Huerta A, Gutiérrez E, Gutiérrez-Solís E, et al. Renoprotective effects of mineralocorticoid receptor blockers in patients with proteinuric kidney diseases. Nephrol Dial Transplant. 2013; 28(2):405-12. 
61 Swan JT, Riche DM, Riche KD, Majithia V. Systematic review and meta-analysis of immunosuppressant therapy clinical trials in membranous lupus nephritis. J Investig Med. 2011;59(2):246-58.

62 Miyakis S, Lockshin MD, Atsumi T, Branch DW, Brey RL, Cervera R, et al. International consensus statement on an update of the classification criteria for definite antiphospholipid syndrome (APS). J Thromb Haemost. 2006;4(2):295-306.

63 Amigo MC. Kidney disease in antiphospholipid syndrome. Rheum Dis Clin North Am. 2006;32(3):509-22.

64 Fakhouri F, Noël LH, Zuber J, Beaufils H, Martinez F, Lebon P, et al. The expanding spectrum of renal diseases associated with antiphospholipid syndrome. Am J Kidney Dis. 2003;41(6):1205-11.

65 Amigo MC, Garcia-Torres R, Robles M, Bochicchio T, Reyes PA. Renal involvement in primary antiphospholipid syndrome. J Rheumatol. 1992;19(8):1181-5.

66 Cheunsuchon B, Rungkaew P, Chawanasuntorapoj R, Pattaragarn A, Parichatikanond P. Prevalence and clinicopathologic findings of antiphospholipid syndrome nephropathy in Thai systemic lupus erythematosus patients who underwent renal biopsies. Nephrology. 2007;12(5):474-80.

67 Moroni G, Ventura D, Riva P, Panzeri P, Quaglini S, Banfi G, et al. Antiphospholipid antibodies are associated with an increased risk for chronic renal insufficiency in patients with lupus nephritis. Am J Kidney Dis. 2004; 43(1):28-36.

68 Galindo M, Gonzalo E, Martinez-Vidal MP, Montes S, Redondo N, Santiago B, et al. Immunohistochemical detection of intravascular platelet microthrombi in patients with lupus nephritis and anti-phospholipid antibodies. Rheumatology. 2009;48(8):1003-7.

69 Gonzalo E, Toldos O, Martínez-Vidal MP, Ordoñez MC, Santiago B, Fernández-Nebro $\mathrm{A}$, et al. Clinicopathologic correlations of renal microthrombosis and inflammatory markers in proliferative lupus nephritis. Arthritis Res Ther. 2012;14(3):R126.

70 Gerhardsson J, Sundelin B, Zickert A, Padyukov L, Svenungsson E, Gunnarsson I. Histological antiphospholipid-associated nephropathy versus lupus nephritis in patients with systemic lupus erythematosus: an observational cross-sectional study with longitudinal follow-up. Arthritis Res Ther. 2015;17:109.

71 Ritis K, Doumas M, Mastellos D, Micheli A, Giaglis S, Magotti P, et al. A novel C5a receptor-tissue factor cross-talk in neutrophils links innate immunity to coagulation pathways. J Immunol. 2006;177(7):4794-802.

72 Ikeda K, Nagasawa K, Horiuchi T, Tsuru T, Nishizaka H, Niho Y. C5a induces tissue factor activity on endothelial cells. Thromb Haemost. 1997;77(2):394-8.
73 Foreman KE, Vaporciyan AA, Bonish BK, Jones ML, Johnson KJ, Glovsky MM, et al. C5a-induced expression of P-selectin in endothelial cells. J Clin Invest. 1994;94(3):114755.

74 Grosso G, Vikerfors A, Woodhams B, Adam M, Bremme K, Holmström M, et al. Thrombin activatable fibrinolysis inhibitor (TAFI): a possible link between coagulation and complement activation in the antiphospholipid syndrome (APS). Thromb Res. 2017;158: $168-73$.

75 Lim W, Crowther MA, Eikelboom JW. Management of antiphospholipid antibody syndrome: a systematic review. JAMA. 2006; 295(9):1050-7.

76 Wong T, Goral S. Lupus nephritis and Kidney transplantation: where are we today? Adv Chronic Kidney Dis. 2019;26(5):313-22.

77 Ames PR, Merashli M, Bucci T, Gentile F, Delgado-Alves J. Antiphospholipid antibodies and renal transplant: a systematic review and meta-analysis. Semin Arthritis Rheum. 2019;48(6):1041-52.

78 Jorgenson MR, Descourouez JL, Singh T, Astor BC, Panzer SE. Malignancy in renal transplant recipients exposed to cyclophosphamide prior to transplantation for the treatment of native glomerular disease. Pharmacotherapy. 2018;38(1):51-7.

79 Contreras G, Mattiazzi A, Guerra G, Ortega LM, Tozman EC, Li H, et al. Recurrence of lupus nephritis after kidney transplantation. J Am Soc Nephrol. 2010;21(7):1200-7.

80 Zhou T, Zhang X, Lin W, Lin S. Multitarget therapy: an effective and safe therapeutic regimen for lupus nephritis. J Pharm Pharm Sci. 2019;22(1):365-75

81 Kattah AG, Garovic VD. Pregnancy and lupus nephritis. Semin Nephrol. 2015;35(5):48799.

82 Smyth A, Radovic M, Garovic VD. Women, kidney disease, and pregnancy. Adv Chronic Kidney Dis. 2013;20(5):402-10.

83 Smyth A, Oliveira GH, Lahr BD, Bailey KR, Norby SM, Garovic VD. A systematic review and meta-analysis of pregnancy outcomes in patients with systemic lupus erythematosus and lupus nephritis. Clin J Am Soc Nephrol. 2010;5(11):2060-8

84 Powe CE, Levine RJ, Karumanchi SA. Preeclampsia, a disease of the maternal endothelium: the role of antiangiogenic factors and implications for later cardiovascular disease. Circulation. 2011;123(24):2856-69.

85 Lynch A, Murphy J, Gibbs R, Levine R, Giclas $\mathrm{P}$, Salmon J, et al. The interrelationship of complement-activation fragments and angiogenesis-related factors in early pregnancy and their association with pre-eclampsia: complement, angiogenesis, pre-eclampsia and obesity. BJOG. 2010;117:456-62.
86 Kim MY, Buyon JP, Guerra MM, Rana S, Zhang D, Laskin CA, et al. Angiogenic factor imbalance early in pregnancy predicts adverse outcomes in patients with lupus and antiphospholipid antibodies: results of the PROMISSE study. Am J Obstet Gynecol. 2016;214(1):108-e14.

87 Herraiz García I, Jiménez L, Elena A, Arriaga G, Isabel P, Escribano Abad D, et al. Doppler de arterias uterinas y marcadores angiogéni$\cos$ (sFlt-1/PlGF): futuras implicaciones para la predicción y el diagnóstico de la preeclampsia. Diagnóstico Prenat. 2011;22:32-40.

88 Redman CW, Sargent IL. Immunology of preeclampsia. Am J Reprod Immunol. 2010; 63(6):534-43.

89 Zeher M, Doria A, Lan J, Aroca G, Jayne D, Boletis I, et al. Efficacy and safety of entericcoated mycophenolate sodium in combination with two glucocorticoid regimens for the treatment of active lupus nephritis. Lupus. 2011;20(14):1484-93.

90 Ruiz-Irastorza G, Ugarte A, Saint-Pastou Terrier C, Lazaro E, Iza A, Couzi L, et al. Repeated pulses of methyl-prednisolone with reduced doses of prednisone improve the outcome of class III, IV and V lupus nephritis: an observational comparative study of the lupusCruces and lupus-Bordeaux cohorts. Autoimmun Rev. 2017;16(8):826-32.

91 Götestam Skorpen C, Hoeltzenbein M, Tincani A, Fischer-Betz R, Elefant E, Chambers $\mathrm{C}$, et al. The EULAR points to consider for use of antirheumatic drugs before pregnancy, and during pregnancy and lactation. Ann Rheum Dis. 2016;75(5):795-810.

92 Ruiz-Irastorza G, Ramos-Casals M, Brito-Zeron P, Khamashta MA. Clinical efficacy and side effects of antimalarials in systemic lupus erythematosus: a systematic review. Ann Rheum Dis. 2010;69(1):20-8.

93 Monova D, Belovezhdov N, Altunkova I, Monov S. Intravenous immunoglobulin G in the treatment of patients with chronic glomerulonephritis: clinical experience lasting 15 years. Nephron. 2002;90(3):262-6.

94 Østensen M, Khamashta M, Lockshin M, Parke A, Brucato A, Carp H, et al. Anti-inflammatory and immunosuppressive drugs and reproduction. Arthritis Res Ther. 2006; 8(3):209.

95 Martínez López JA, García Vivar ML, Cáliz R, Freire M, Galindo M, Hernández MV, et al. Recommendations for the evaluation and management of patients with rheumatic autoimmune and inflammatory diseases during the reproductive age, pregnancy, postpartum and breastfeeding. Reumatol Clin. 2017; 13(5):264-81.

96 Sangle SR, Lutalo PM, Davies RJ, Khamashta MA, D'Cruz DP. B-cell depletion therapy and pregnancy outcome in severe, refractory systemic autoimmune diseases. J Autoimmun. 2013;43:55-9. 
97 Kronbichler A, Brezina B, Quintana LF, Jayne DR. Efficacy of plasma exchange and immunoadsorption in systemic lupus erythematosus and antiphospholipid syndrome: a systematic review. Autoimmun Rev. 2016;15(1): 38-49.

98 Flint J, Panchal S, Hurrell A, van de Venne M, Gayed M, Schreiber K, et al. BSR and BHPR guideline on prescribing drugs in pregnancy and breastfeeding - part II: analgesics and other drugs used in rheumatology practice. Rheumatology. 2016;55:1698-702.

99 Fanouriakis A, Kostopoulou M, Alunno A, Aringer M, Bajema I, Boletis JN, et al. 2019 update of the EULAR recommendations for the management of systemic lupus erythematosus. Ann Rheum Dis. 2019;78(6):736-45.
100 Tamirou F, D’Cruz D, Sangle S, Remy P, Vasconcelos C, Fiehn C, et al. Long-term follow-up of the MAINTAIN Nephritis Trial, comparing azathioprine and mycophenolate mofetil as maintenance therapy of lupus nephritis. Ann Rheum Dis. 2016;75(3): 526-31.

101 Fanouriakis A, Bertsias G. Changing paradigms in the treatment of systemic lupus erythematosus. Lupus Sci Med. 2019;6(1): e000310.

102 Parikh SV, Almaani S, Brodsky S, Rovin BH. Update on lupus nephritis: core curriculum 2020. Am J Kidney Dis. 2020 Mar 24(19): 31170-9.
103 Fanouriakis A, Kostopoulou M, Cheema K, Anders HJ, Aringer M, Bajema I, et al. 2019 update of the Joint European League against Rheumatism and European Renal Association-European Dialysis and Transplant Association (EULAR/ERA-EDTA) recommendations for the management of lupus nephritis. Ann Rheum Dis. 2020 Jun;79(6): 713-23.

104 Jayne D, Bajema IM. "In my beginning is my end": usefulness of repeat kidney biopsies in lupus nephritis. Kidney Int. 2020;97(1):279.

105 Bomback AS. An update on therapies for proliferative lupus nephritis: how certain can we be about the evidence? Am J Kidney Dis. 2018;72(5):758-60. 